Check for updates

Cite this: Chem. Commun., 2019, 55, 9817

Received 21st May 2019,

Accepted 22nd July 2019

DOI: $10.1039 / c 9 c c 03941 d$

rsc.li/chemcomm

\section{The key to controlling the morphologies of quantum nanocrystals: spherical carborane ligands $\dagger$}

\author{
Abhishek Saini, Arpita Saha, Clara Viñas (D) and Francesc Teixidor (D) *
}

\begin{abstract}
By minor structure modification of spherical carborane ligands, in a similar synthetic procedure, large morphological changes are produced in Quantum Nanocrystals (QNCs). The spheres are icosahedral $\mathrm{C}_{2} \mathrm{~B}_{10} \mathrm{H}_{12}$ molecules with binding sites on the carbon, and the QNCs produced are Quantum Dots, Rods, Rings and Tetrapods. These QNCs demonstrated high stability and impeccable emissive properties for more than a year.
\end{abstract}

Carboranes have geometrical figures that correspond to deltahedra having atoms only in the periphery, the more stable being the icosahedron. ${ }^{1 a, b}$ The icosahedral boron clusters, $\left[\mathrm{B}_{12} \mathrm{H}_{12}\right]^{2-},\left[\mathrm{CB}_{11} \mathrm{H}_{12}\right]^{-}, 1,2-\mathrm{C}_{2} \mathrm{~B}_{10} \mathrm{H}_{12}$ or $1,7-\mathrm{C}_{2} \mathrm{~B}_{10} \mathrm{H}_{12}$ have spherical structures and are more readily available than the geometrically similar hydrocarbon dodecahedrane $\left(\mathrm{C}_{20} \mathrm{H}_{20}\right)$, which is very difficult to synthesize. ${ }^{1 c}$ Contrastingly, the icosahedral boranes can be synthesized in a single step. ${ }^{1 d}$ Their properties make them very attractive for material's science, particularly by the monolayer mode of packing when placed on a surface, either hexagonal or square. ${ }^{1 e}$ Placing these spheres in closed packing generates openings which facilitate the movement of ions and molecules. ${ }^{2 a}$ The properties of the carboranyl spheres were further used to link them to the binding sites of Quantum Dots (QDs) to produce CdSe QDs with a novel core canopy architecture. $^{2 b}$

The relevance of ligands in the synthesis of Quantum Nanocrystals (QNCs) is well established. ${ }^{3}$ The ligands, which are commonly hydrophobic organic molecules, prevent the formation of a bulk semiconductor material by coordination with the unsaturated metal atoms on the surface of QDs. ${ }^{4}$ The size of the quantum dot is determined by the crystal growth time. ${ }^{4}$ Quantum Rods $\left(\mathrm{QR}_{\mathrm{ods}}\right)^{5}$ and Quantum Rings $(\mathrm{QRs})^{6}$ have also been synthesized using colloidal procedures and using

Institut de Ciencia de Materials de Barcelona, Campus de la UAB, Bellaterra, o8193, Spain.E-mail: teixidor@icmab.es

$\dagger$ Electronic supplementary information (ESI) available: The structure of different ligands, schematic of synthesis and TEM, STEM and HRTEM images, UV-vis absorbance, IR, EELS, EDX spectra, electron diffraction patterns, ICP-MS analysis and detailed experimental procedure. See DOI: 10.1039/c9cc03941d ligands as capping agents for shape modification, though to date these procedures are highly complex and can't match the ease with which these QRs and $\mathrm{QR}_{\mathrm{ods}}$ can be synthesized by physical methods. The electronic and optoelectronic properties of nanocrystals depend strongly on the shape of the nanostructure. ${ }^{7}$ In this regard, QRs are unique nanostructures with unique properties because of their circular geometry. ${ }^{8}$ To study these fascinating nano-structures, efficient methods to produce QRs are necessary. But for one reported case of colloidal synthesis that requires etching, ${ }^{6}$ QRs are currently made by physical methods using electron beam lithography, and ion beam milling. ${ }^{9}$ Distinct to $\mathrm{QRs}, \mathrm{QR}_{\mathrm{ods}}$ are of great interest due to their elongated lengths providing a number of advantages over the traditional QDs and their own set of unique properties. ${ }^{10}$ These make $\mathrm{QR}_{\text {ods }}$ highly desirable in the field of applications like in fluorescent labels and markers but they experience low throughput as QRs when prepared using physical methods.

To produce different morphologies of QNCs following a colloidal procedure, we relied on unconventional ligands with spheres having an appended coordinating site, i.e., carboranyl ligands. The reason behind this is that spheres can ideally pack in a compact way with a hexagonal or square arrangement, thus influencing the inner core by the outer sphere packing. Here, we have used a standard colloidal synthetic method, ${ }^{11}$ emphasizing on the ligand used, to synthesize QNCs with different morphologies, and high throughput. QDs, $\mathrm{QR}_{\text {ods }}$, QRs, and Quantum Tetrapods (QTPs) have been produced. Seven carborane derivatives ${ }^{12}$ have been studied in this work as depicted in Fig. S1 (ESI $\dagger$ ), and described in Table 1 . The synthetic procedure used is a modified version of a previously reported one. ${ }^{13}$

Our approach assumed that the compactness of the arranged spherical carborane units would create curvatures. The concept is schematically shown in Fig. 1 . The scheme in Fig. 1(i) implies a perfect matching of the sphere's van der Waals diameter and the covalent diameter of the Cd-Se. However, if this matching is good, but not perfect, as the two diameters are $656 \mathrm{pm}$ vs. $528 \mathrm{pm}$ respectively, then the only 
Table 1 This table depicts the different shapes formed at the quantum regime by the changing of the carborane ligands

\begin{tabular}{|c|c|c|}
\hline $\begin{array}{l}\text { Carborane derivative acting as } \\
\text { a capping agent }\end{array}$ & $\begin{array}{l}\text { Quantum- } \\
\text { nanocrystal } \\
\text { morphology }\end{array}$ & $\begin{array}{l}\text { Colors of } \\
\text { emission }\end{array}$ \\
\hline meta-Carboranethiol & QDs & Blue to yellow \\
\hline ortho-Carboranethiol & $\mathrm{QR}_{\mathrm{ods}}$ & Blue to yellow \\
\hline meta-Carboranedithiol & $\mathrm{QR}_{\text {ods }}$ & Shades of yellow \\
\hline 1-Methyl-ortho-2-carboranethiol & QRs & Blue to yellow \\
\hline meta-Carboranephosphinic acid & QRs & Blue and green \\
\hline meta-Carboranediphosphinic acid & QRs & Blue and green \\
\hline meta-Carboranecarboxylic acid & QTPs & Green and yellow \\
\hline
\end{tabular}

(i)

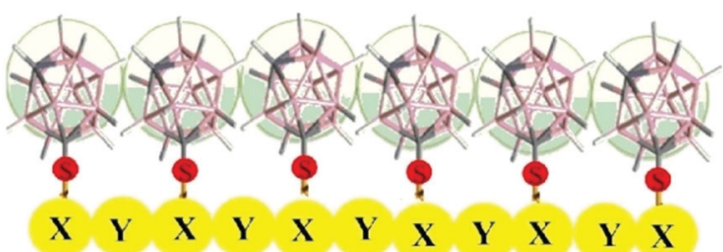

(ii)

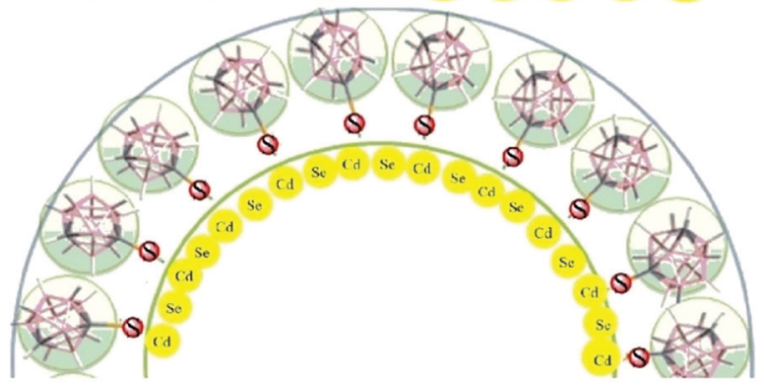

Fig. 1 The schematic of the sphere concept for different geometries. (i) The case for an ideal matching between the diameter of the sphere and the diameter of the $\mathrm{X}-\mathrm{Y}$ unit. (ii) The case for a mismatch, as is the case of the carborane diameter and the CdSe covalent diameter.

way for them to fit with each other would be through the model shown in Fig. 1(ii), i.e., the two concentric circles.

To perform the synthesis, it was needed to carefully control the temperature and its ramps. If the temperature is not ramped up and increased stepwise, independently of the carborane used, perfectly spherical shaped QDs are produced. These QDs have sizes in the range of 2 to $3 \mathrm{~nm}$ in diameter with varying colours depending on the elapsed time of pipetting of the samples. Upon incorporating a sudden increase in temperature for the last $40-50{ }^{\circ} \mathrm{C}$, we observe the formation of differently shaped NCs, depending upon the ligand used. It must be pointed out that meta-carboranethiol always produces spherical QDs irrespective of the sudden temperature increase. Table 1 lists the different morphologies formed with their respective capping agent.

The abrupt rise in temperature from $150{ }^{\circ} \mathrm{C}$ to $195{ }^{\circ} \mathrm{C}$ has already been reported as necessary to produce $\mathrm{QR}_{\text {ods }}$ with TOPO/Oleic Acid. ${ }^{14}$ We suggest that this abrupt temperature change induces the formation of nanobubbles that become the template to generate different morphologies other than the traditional QDs, given the appropriate ligands. After the synthesis procedure was completed, efforts were made to completely
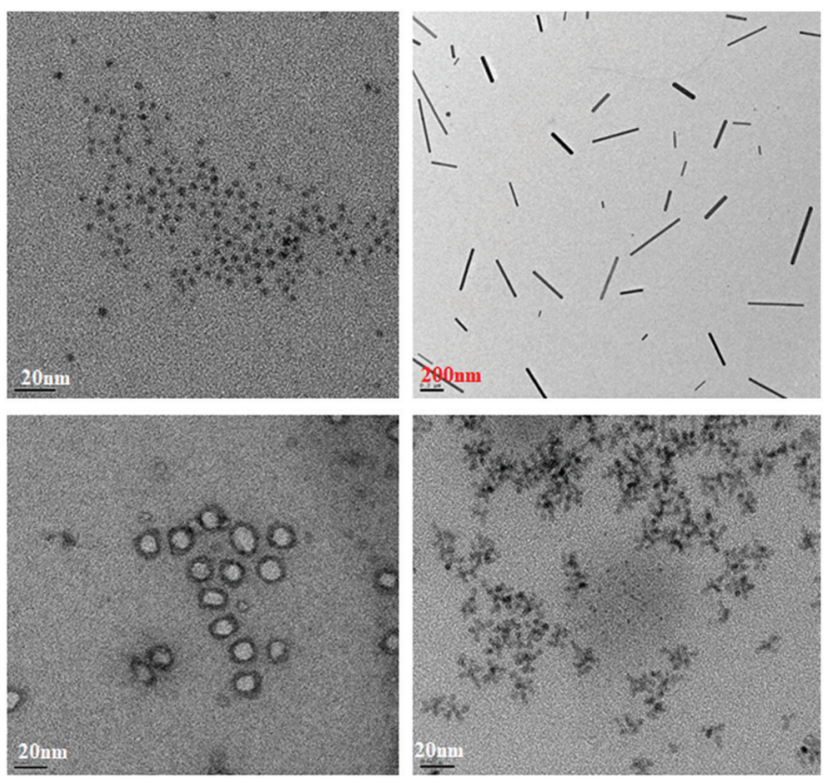

Fig. 2 The different shapes are shown in this figure. The top left corner shows the QDs, the top right shows the $\mathrm{QR}_{\text {ods, }}$ the bottom left shows the QRs and the bottom right shows the QTDs.

isolate the QNCs hence produced, from their colloidal suspension. After unsuccessful attempts with the use of PTFE microfilters, a modicum of success was achieved by using ethanol to highly dilute the colloidal suspension and then centrifuging it as seen in Fig. S2a in the ESI. $\dagger$

Of particular interest amongst these QNCs is the formation of QRs and QTPs, ${ }^{15}$ the colloidal synthesis of which is extremely rare and certainly has never been achieved before with such ease in a one-step synthesis. The different QNCs formed are shown in Fig. 2, while Fig. S2b in the ESI $\dagger$ depicts the facile growth of the QRs after Se addition.

The TEM, STEM and HRTEM images of the QNCs are shown in Fig. S3 and S4, ESI. $†$ The existence of B, Cd, Se and P or S in these QNCs was proven by spectroscopic techniques. The presence of Boron indicating carborane was detected by EELS

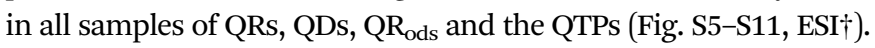
The B-H band appears around $2500 \mathrm{~cm}^{-1}$ near the range of S-H, but the disappearance of the S-H band in the QDs has already been reported $^{16}$ (Fig. S12-S14, ESI $\dagger$ ). For QRs, the ratio Cd:Se is $2: 1$. This is not unexpected as traditionally in most QDs the Cd: Se ratio is skewed in favour of $\mathrm{Cd}$, but not so much as to reach a welldefined 2:1. The EDX of all QNCs shows a ratio of Cd:Se as $2: 1$, but for the QTPs that is of $3: 2$. This ratio of $\mathrm{Cd}: \mathrm{Se}$ remained constant over 5 months (Fig. S16-S22, ESI $\dagger$ ). The ICP-MS analysis for the samples also showed the ratio of Cd:Se as 2:1 (Table S1, ESI $\dagger$ ). For QRs generated with meta-carborane phosphinic acid and meta-carboranediphosphinic acid, the EDX ratio of elements $\mathrm{Cd}: \mathrm{Se}: \mathrm{P}$ is $2: 1: 4$.

The importance of ligands in controlling morphologies of NCs in colloidal synthesis has been reported ${ }^{17}$ but the mechanism of this is still not clear. Viewed with a certain perspective, one could envisage similarities between the sphere-to-rod transitions of non-ionic surfactant micelles in aqueous solution ${ }^{18}$ 
and the QDs to $\mathrm{QR}_{\mathrm{ods}}$ possible transition that is considered in this work. However, they are not related. In the first one, the transition is due to an increasing concentration of the same surfactant going through two CMC; in the case reported here all syntheses are carried out at the same concentration of $\mathrm{Cd}$ and Se precursors and the distinct carboranes. Furthermore, the solvent and temperature and temperature ramps are alike in the different processes. The only differences are the carboranes with their distinct ligating sites and non-binding substituents. Thus, it is our hypothesis that is the interplay of the hydrophobic interactions between carborane units, the electronically minor but sterically relevant carborane substituents, the sphere packings and the consequences of the mismatch described earlier that lead to the formation of these geometric features. We do not discard, however, the assistance of the nanobubble template effect which would be originated by the sudden temperature rise. This is conjectural but may be an explanation to account for the necessity of abrupt temperature increase to generate different QNCs, as opposed to the traditional QDs if the abrupt temperature rise does not take place. Electron diffraction studies confirmed the synthesis of CdSe materials with a hexagonal phase (Fig. S23, ESI $\dagger$ ), in all the QNCs. The $\mathrm{QR}_{\text {ods }}$ and QTPs had a more crystalline diffraction pattern while the QRs and the QDs had a more amorphous diffraction pattern. Fig. S26 (ESI $\dagger$ ) shows the mean size of the QDs, $\mathrm{QR}_{\mathrm{ods}}$ and QRs formed. They are all well within the quantum regime of sizes. To substantiate the relevance of the spheres in the formation of QNCs we compared the carboranethiol with adamantanethiol by using the same conditions of synthesis leading to QNCs. The result was the formation of QDs with a weak fluorescence. No other morphology was found proving the necessity of the carborane ligands in producing these novel structures (Fig. S25a and b, ESI $\dagger$ ).

The fluorescence intensities of the QNCs produced using carborane derivatives are far superior to the QDs commonly synthesized in an organic medium capped with TOPO and TOP. The carborane capped QNCs are fluorescent and maintain their lifetime of fluorescence for up to a year as tested. The intensity of $\mathrm{QRs}, \mathrm{QR}_{\mathrm{ods}}$ and QDs are measured using filters, which only allow for $1 \%$ of the total intensity of emission to be recorded, as the intensity of these QNCs was too high to be recorded by the arbitrary units of the fluorometer. The fluorescence colours shown by these QNCs vary greatly, depending upon the different ligands used and also upon the time of pipetting out of the sample from the reaction mixture. Based upon the time elapsed of the reaction after Se addition, the QNCs capped by the same ligand can show different colours (Table 1).

The QRs with 1-methyl-ortho-2-carboranethiol emit colours from blue to yellow suggesting a facile growth process with time (see Fig. S1b, ESI $\dagger$ ), while the QRs with meta-carborane phosphinic acid and meta-carboranediphosphinic acid show mainly green and bluish colour that suggests a less favourable growth process. The QDs made with meta-carboranethiol emit different shades ranging from green to yellow. The $\mathrm{QR}_{\mathrm{ods}}$ capped with the ortho-carboranethiol emit colours from blue to yellow while the $\mathrm{QR}_{\mathrm{ods}}$ capped with meta-carboranedithiol emit shades of yellow. In terms of luminescence, the most curious property is shown by the QTPs capped with meta-carboranecarboxylic acid. While the emission of the rest of the QNCs remains constant after being pipetted out, irrespective of their storage time, the QTPs show variance in colours even after they have been pipetted out of the reaction mixture. Their emission keeps on changing over the course of time in storage. They emit green colour on the day of the reaction, which changes to yellow after 2 months and to orange after 5 months. This change in colour would normally suggest that the QTPs keep growing in size over time or aggregating, but TEM images show no increase in size even after 5 months of storage. The EDX analysis also showed that the chemical composition of these QTPs stays constant over 5 months (Fig. S22, ESI $\dagger$ ). The change in colour seems to

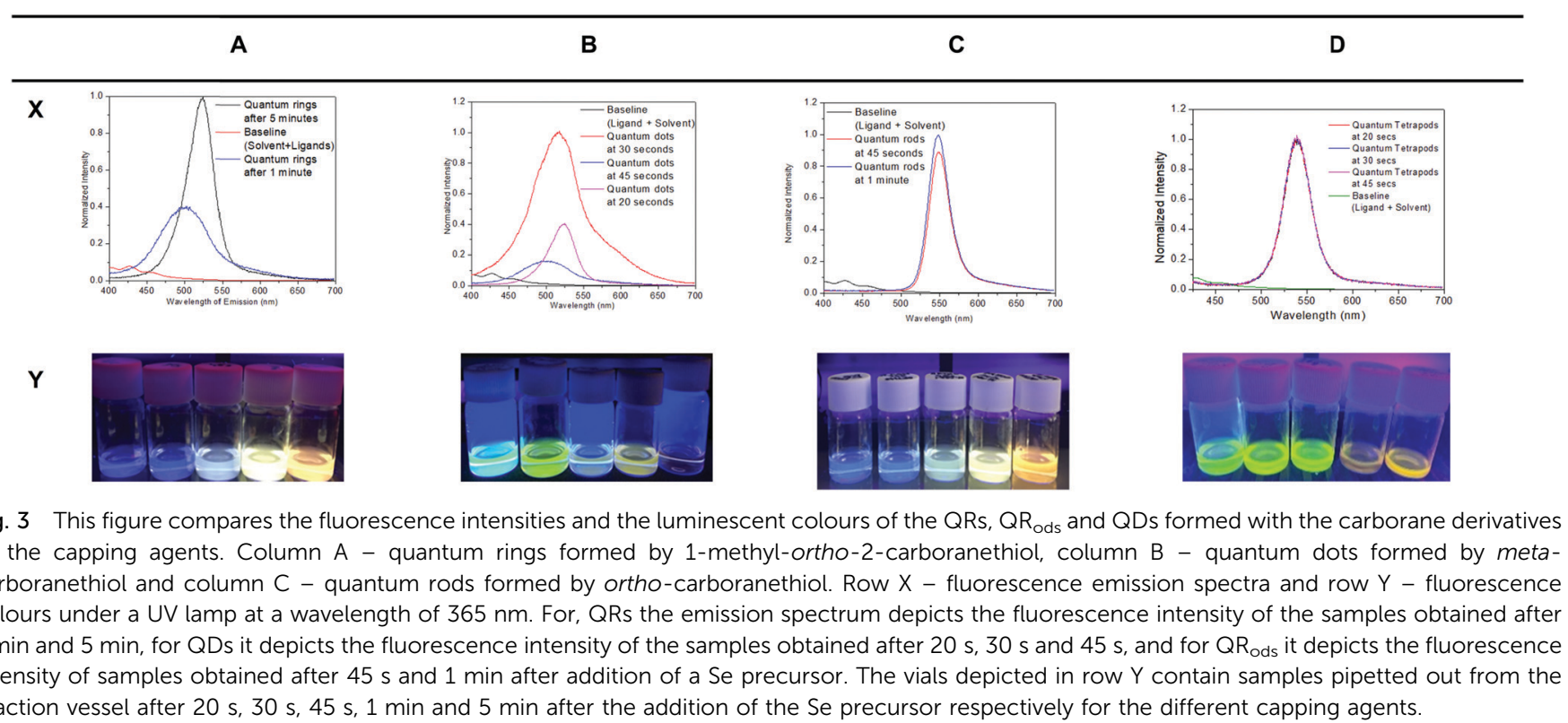


be independent of the size and chemical composition. Our tentative explanation is that different phases are generated as a result of the ageing of the samples which leads to this colour change. In support of this, we found that the recently formed QTPs have the hexagonal wurtzite structure while the older QTPs show a cubic zinc blende phase (Fig. S23b and S24a, ESI $\dagger$ ). The fluorescence intensities are further represented in Fig. 3, with baseline intensity already accounted for. The absorption spectra are recorded and they lie within the 300 to $400 \mathrm{~nm}$ range with maximum absorption around $360 \mathrm{~nm}$ (Fig. S27-S29, ESI $\dagger$ ).

In summary, this paper demonstrates the ease with which we can control the morphology of various QNCs, all the while following the same colloidal synthesis procedure, by incorporating spherical carborane ligands as capping agents. The QNCs hence produced are all highly stable, and show high fluorescence intensities. The use of icosahedral carboranes of formula $\mathrm{C}_{2} \mathrm{~B}_{10} \mathrm{H}_{12}$ for the synthesis of QRs, $\mathrm{QR}_{\mathrm{ods}}$ and QTPs is unprecedented and highlights the relevance of the spherical ligands. This synthetic procedure can facilitate a shift in focus from trying to find a viable synthesis procedure for morphologies such as QRs and QTPs, towards studying them and their properties in further detail and producing them in sufficient quantities for use in novel applications.

This work has been supported by the Spanish Ministerio de Economia y Competitividad (CTQ2016-75150-R) and Generalitat de Catalunya (2014/SGR/149). A. Saha thanks Generalitat de Catalunya for FPI predoctoral grant (2016FI_B 00214). A. Saini and $\mathrm{A}$. Saha are enrolled in the $\mathrm{PhD}$ in Chemistry program of UAB.

\section{Conflicts of interest}

There are no conflicts to declare.

\section{Notes and references}

1 (a) J. Poater, M. Solà, C. Viñas and F. Teixidor, Angew. Chem., Int. Ed., 2014, 53, 12191-12195; (b) J. Poater, M. Solà, C. Viñas and F. Teixidor, Chem. - Eur. J., 2016, 22, 7437-7443; (c) L. A. Paquette, R. J. Ternansky, D. W. Balogh and G. Kentgen, J. Am. Chem. Soc., 1983, 105, 4446-4450; (d) R. Caputo, S. Garroni, D. Olid, F. Teixidor, S. Suriñach and M. D. Baro, Phys. Chem. Chem. Phys., 2010, 12, 15093; (e) R. N. Grimes, Carboranes, Elsevier, Oxford, 3rd edn, 2016.

2 (a) A. M. Cioran, A. D. Musteti, F. Teixidor, Ž. Krpetić, I. A. Prior, Q. He, C. J. Kiely, M. Brust and C. Viñas, J. Am. Chem. Soc., 2012, 134, 212-221; (b) A. Saha, E. Oleshkevich, C. Vinas and F. Teixidor, Adv. Mater., 2017, 29, 1704238.
3 (a) A. P. Alivisatos, W. Gu and C. Larabell, Annu. Rev. Biomed. Eng., 2005, 7, 55-76; (b) J. Weng and J. Ren, Curr. Med. Chem., 2006, 13, 897-909.

4 S. N. Raja, A. C. K. Olson, K. Thorkelsson, A. J. Luong, L. Hsueh, G. Chang, B. Gludovatz, L. Lin, T. Xu, R. O. Ritchie and A. P. Alivisatos, Nano Lett., 2013, 13, 3915-3922.

5 (a) A. Wolcott, R. C. Fitzmorris, O. Muzaffery and J. Z. Zhang, Chem. Mater., 2010, 22, 2814-2821; (b) A. E. Saunders, F. Shieh and B. A. Korgel, J. Phys. Chem. B, 2005, 109, 8538-8542; (c) K. Manthiram, B. J. Beberwyck, D. V. Talapin and A. P. Alivisatos, J. Visualized Exp., 2013, 82, 50731.

6 I. Fedin and D. V. Talapin, J. Am. Chem. Soc., 2016, 138, 9771-9774.

7 K. D. Gilroy, A. Ruditskiy, H. C. Peng, D. Qin and Y. Xia, Chem. Rev., 2016, 116, 10414-10472.

8 (a) C. Tong, S. Yoon and L. Wang, Nanoscale Res. Lett., 2012, 7, 520; (b) V. E. Demidov, S. Urazhdin, G. de Loubens, O. Klein, V. Cros, A. Anane and S. O. Demokritov, Phys. Rep., 2017, 673, 1-31; (c) A. Fuhrer, S. Lüscher, T. Ihn, T. Heinzel, K. Ensslin, W. Wegscheider and M. Bichler, Nature, 2001, 413, 822-825; (d) W. C. Tan and J. C. Inkson, Phys. Rev. B: Condens. Matter Mater. Phys., 1999, 60, 5626-5635; (e) V. I. Klimov, S. A. Ivanov, J. Nanda, M. Achermann, I. Bezel, J. A. McGuire and A. Pirystinski, Nature, 2007, 447, 441-446; $(f)$ G. O. De Sousa, D. R. Da Costa, A. Chaves, G. A. Farias and F. M. Peeters, Phys. Rev. B, 2017, 95, 20514.

9 (a) A. Lorke, R. J. Luyken, M. Fricke, J. P. Kotthaus, G. MedeirosRibeiro, J. M. Garcia and P. M. Petroff, Microelectron. Eng., 1999, 47, 95-99; (b) A. Lorke, R. J. Luyken, A. O. Govorov, J. P. Kotthaus, J. M. Garcia and P. M. Petroff, Phys. Rev. Lett., 2000, 84, 2223-2226; (c) D. Haft, F. Bickel, A. Lorke, K. Karrai, J. M. Garcia, W. Schoenfeld and P. M. Petroff, Nature, 2000, 405, 8-11; (d) C. Somaschini, S. Bietti, N. Koguchi and S. Sanguinetti, Nanotechnology, 2011, 22, 185602; (e) C. Somaschini, S. Bietti, S. Sanguinetti, N. Koguchi and A. Fedorov, Nanotechnology, 2010, 21, 125601.

10 (a) L. Li, J. Hu, W. Yang and A. P. Alivisatos, Nano Lett., 2001, 1, 349-351; (b) J. Hu, L. S. Li, W. Yang, L. Manna, L. W. Wang and A. P. Alivisatos, Science, 2001, 292, 2060-2063; (c) A. Shabaev and A. L. Efros, Nano Lett., 2004, 4, 1821-1825; (d) H. Htoon, J. A. Hollingworth, A. V. Malko, R. Dickerson and V. I. Klimov, Appl. Phys. Lett., 2003, 82, 4776-4778; (e) E. Rothenberg, M. Kazes, E. Shaviv and U. Banin, Nano Lett., 2005, 5, 1581-1586.

11 (a) B. O. Dabbousi, J. Rodriguez-Viejo, F. V. Mikulec, J. R. Heine, H. Mattoussi, R. Ober, K. F. Jensen and M. G. Bawendi, J. Phys. Chem. B, 1997, 101, 9463-9475; (b) I. L. Medintz, H. T. Uyeda, E. R. Goldman and H. Mattoussi, Nat. Mater., 2005, 4, 435.

12 (a) C. Viñas, R. Benakki, F. Teixidor and J. Casabó, Inorg. Chem., 1995, 34, 3844-3845; (b) E. Oleshkevich, F. Teixidor, D. ChoquesilloLazarte, R. Sillanpää and C. Viñas, Chem. - Eur. J., 2016, 22, 3665-3670; (c) M. Fontanet, A. R. Popescu, X. Fontrodona, M. Rodriguez, I. Romero, F. Teixidor, C. Viñas, N. A. Alcalde and E. Ruiz, Chem. - Eur. J., 2011, 17, 13217-13229.

13 C. A. Ubani and S. M. Yusof, J. Mod. Educ. Rev., 2011, 1, 63-73.

14 W. J. Baumgardner, Z. Quan, J. Fang and T. Hanrath, Nanoscale, $2012,4,3625$.

15 (a) T. Mokari, E. Rothenburg, I. Popov, R. Costi and U. Banin, Science, 2004, 304, 1787-1790; (b) L. Manna, D. J. Miliron, A. Meisel, E. C. Scher and A. P. Alivisatos, Nat. Mater., 2003, 2, 382-385.

16 A. E. Vikraman, A. R. Jose, M. Jacob and K. G. Kumar, Anal. Methods, 2015, 7, 6791-6798.

17 (a) A. Y. Koposov, CrystEngComm, 2017, 19, 3098-3102; (b) W. W. Yu, Y. A. Wang and X. Peng, Chem. Mater., 2003, 15, 4300-4308.

18 M. Velinova, D. Sengupta, A. V. Tadjer and S. J. Marrink, Langmuir, 2011, 27, 14071-14077. 